REGARDS

SUR L'ECONOMIE ALLEMANDE

BULLETIN ECONOMIQUE DU CRAC

\section{Regards sur l'économie allemande}

Bulletin économique du CIRAC

$107 \mid 2012$

Varia

\title{
Le difficile rapport des Allemands à la liberté
}

\section{Thomas Petersen}

Traducteur : Isabelle Bourgeois

\section{(2) OpenEdition}

Journals

Édition électronique

URL : http://journals.openedition.org/rea/4494

DOI : $10.4000 /$ rea.4494

ISBN : 978-2-8218-1424-0

ISSN : 1965-0787

Éditeur

CIRAC

Édition imprimée

Date de publication : 20 décembre 2012

Pagination : 15-33

ISSN : 1156-8992

\section{Référence électronique}

Thomas Petersen, "Le difficile rapport des Allemands à la liberté », Regards sur l'économie allemande

[En ligne], 107 | décembre 2012, mis en ligne le 01 décembre 2014, consulté le 03 mai 2019. URL

http://journals.openedition.org/rea/4494 ; DOI : 10.4000/rea.4494 


\section{Le difficile rapport des Allemands à la liberté}

\section{Thomas Petersen}

\section{Présentation}

"J'aimerais rappeler aujourd'hui un élément fondamental, un levier de la construction européenne, à savoir la liberté qui, seule, permet une existence pacifique et prospère. La liberté doit toujours être défendue sous toutes ses formes : la liberté d'expression, de la presse, de religion et de réunion. Sans la liberté, l'Etat de droit n'existe pas. Sans la liberté, il n'y a ni diversité ni tolérance. La liberté est le socle de l'Europe unie et déterminée ". Ce credo figure au début du discours tenu par la chancelière Angela Merkel devant le Parlement européen le 8 novembre 2012.

Le concept de liberté est d'actualité à plusieurs égards. Aussi bien dans le contexte mondial de l'affrontement entre régimes démocratiques et liberticides que dans le contexte européen des débats sur la poursuite de l'intégration et les réponses à apporter à la crise de la dette et de la compétitivité, où il est toujours associé à celui de responsabilité, assumée ou non. La liberté des Etats membres - leur souveraineté -, en matière de politique économique et budgétaire par exemple, s'accompagne toujours, du moins en théorie et dans le droit communautaire, de son corollaire indissociable qu'est le respect des règles et normes communes, autrement dit : la responsabilité pleinement assumée de l'appartenance à l'UE. A ce niveau politique, Français et Allemands se distinguent par la lecture divergente du sens à accorder à ce concept et à ses deux facettes. Dans le contexte politique français, la thématique de la liberté se manifeste actuellement dans la dialectique entre libéralisme économique et protection sociale ou protection des acquis sociaux. Dans le débat politique allemand, qui semble "ultralibéral " aux yeux de nombre de Français, la controverse sur le rôle de l'Etat dans l'organisation de la société et la préservation de l'équité ou de la justice sociale bat son plein aussi, surtout à l'approche des élections au Bundestag dans moins d'un an. Cette échéance nourrit chez certains le désir de revenir en arrière sur des réformes comme celles de l'Agenda 2010 qui rompaient avec la dérive égalitariste du système de protection sociale pour rétablir le principe fondateur de l'ordre étatique allemand: celui de l'équité des chances, autre acception du principe de liberté. Enfin, dans le contexte de la liberté d'opinion qui caractérise nos démocraties, les récentes controverses, françaises, allemandes, européennes et même mondiales, qui ont jailli à propos d'un certain nombre de caricatures, ont replacé elles aussi au centre de l'intérêt la question de la liberté - sous l'angle de la liberté d'opinion et de la tolérance, cette fois-ci.

Qu'en est-il des Allemands ? Au plan du droit constitutionnel, la RFA est une démocratie solide et mature. Mais qu'en est-il dans la réalité ? Dans son essai "Liberté - un plaidoyer " publié au début de 2012, le président de la République Joachim Gauck constatait que la notion de liberté mature est en perte de vitesse dans une société allemande où la défense des acquis semble actuellement l'emporter. Sa définition de cette liberté mature? " Les citoyens sont des gens qui ont des droits civiques et qui ont le pouvoir de les exercer". Les sondages et enquêtes au long cours réalisés par l'Institut für Demoskopie d'Allensbach et que présente ici l'un de ses responsables sont riches d'enseignements à cet égard: visiblement, les Allemands aussi ont un rapport complexe et difficile avec le principe de liberté...

I. Bourgeois

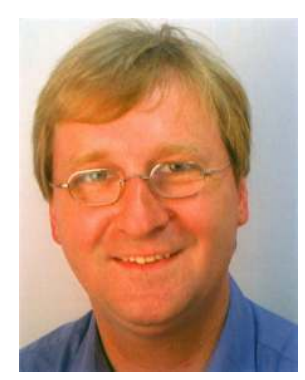

Thomas Petersen, docteur en sciences de la communication, est chercheur à l'Institut für Demoskopie d'Allensbach. Il enseigne également à l'Université de Mayence et préside la World Association for Public Opinion Research (WAPOR). Dans le quotidien F.A.Z., il publie régulièrement les "Allensbach-Analysen" sur l'état de l'opinion. 


\section{Le difficile rapport des Allemands à la liberté}

France et RFA : deux hymnes nationaux...

... très proches...

mais dont la similitude n'est plus perceptible

Tocqueville : l'égalité au service de la liberté

Egalité au sens d'équité des chances $=$ égalité en droit
"Liberté, Egalité, Fraternité ! " Au fil de l'Histoire, nul appel ne s'est imposé avec une telle force que le cri des champs de bataille de la Révolution française. Aujourd'hui encore, les Français, mais aussi les Allemands comme certainement les citoyens de nombre d'autres pays, professent ce credo en vibrant d'émotion. Mais combien d'entre eux en comprennent le sens profond ? Combien, parmi les Allemands qui invoquent ce "Liberté, Egalité, Fraternité » sont conscients qu'ils entonnent la même fanfare lorsqu'ils chantent leur propre hymne national ? Les deux premiers vers de la troisième strophe du Chant des Allemands, qui constitue l'hymne national, sont ainsi formulés : "Unité et droit et liberté pour la patrie allemande!" / "Tendons tous vers cela fraternellement, avec le cœur et la main! »

"Unité et droit et liberté » - il n'y a rien du cri de révolte de 1789 dans ces mots qui évoquent plutôt la sérénité à laquelle aspirent d'honorables citoyens. Et pourtant, le triple accord "Unité et droit et liberté » ne figure pas par hasard dans le Chant des Allemands, mais y a été à n'en pas douter inséré très consciemment, inspiré par le chant révolutionnaire français, voire traduit directement du français. Car le Chant des Allemands aussi est un chant révolutionnaire : il a été écrit en 1841, dans les années d'agitation politique du Vormärz qui précédèrent la révolution citoyenne (bourgeoise) de 1848. L'auteur, le poète August Heinrich Hoffmann von Fallersleben, était un ardent défenseur des droits du citoyen (du bourgeois : Bürger). Et dès lors, mis à part le contraste dans leur tonalité, la seule différence entre le début des hymnes nationaux allemand et français réside dans le concept d'« unité » (Einigkeit), un terme à double sens en allemand. On peut y lire "fraternité " bien sûr, mais il renvoie bien plus certainement encore à l'unité politique d'une Allemagne qui, en 1841, est éclatée entre des douzaines de royautés et principautés, en partie minuscules. Et de fait, la fraternité est évoquée immédiatement après, dans le vers suivant: "fraternellement, avec le cœur et la main ".

Pourquoi alors presque personne ne s'aperçoit que ces deux débuts des hymnes nationaux - l'un dans le registre des braves gens, l'autre empreint de fougue révolutionnaire - sont au fond largement identiques ? Cela tient certainement avant tout au terme "égalité ». Hoffmann le traduit par "droit ». Mais ne s'agit-il pas là de deux notions totalement différentes ? Eh bien, non. Seulement, au cours des 150 ans écoulés, la conscience de cette similitude s'est perdue, enfouie sous une inextricable confusion des sens.

Dans son ouvrage sur la démocratie en Amérique, Alexis de Tocqueville décrit avec fascination comment, au fil des siècles, l'égalité est devenue le principe prédominant de nos sociétés. II le formule ainsi : " $S i$, à partir $d u X{ }^{P}$ siècle, vous examinez ce qui se passe en France de cinquante en cinquante années, au bout de chacune de ces périodes, vous ne manquerez point d'apercevoir qu'une double révolution s'est opérée dans l'état de la société. Le noble aura baissé dans l'échelle sociale, le roturier s'y sera élevé ; l'un descend, l'autre monte. Chaque demi-siècle les rapproche, et bientôt ils vont se toucher. Et ceci n'est pas seulement particulier à la France. De quelque côté que nous jetions nos regards, nous apercevons la même révolution qui se continue dans tout l'univers chrétien. Le développement graduel de l'égalité des conditions est donc un fait providentiel, il en a les principaux caractères : il est universel, il est durable, il échappe chaque jour à la puissance humaine; tous les événements, comme tous les hommes, servent à son développement".

Tocqueville décrit ici l'égalité comme une loi naturelle inéluctable, évoluant en symbiose avec la liberté politique et la liberté de la société. L'égalité est placée au service de la liberté, elle est l'expression de cette dernière, c'est-à-dire de l'émancipation du citoyen et de la fin de la suprématie de la noblesse. Or, et 
Tocqueville est formel là-dessus, il s'agit de l'égalité des conditions. Aujourd'hui, nous dirions équité des chances (Chancengleichheit) ou bien : égalité devant la loi, conjuguée avec la sécurité juridique - en un mot : droit.

Quand a bien pu s'imposer l'idée qu'il ne suffit pas de crééer l'égalité des conditions préalables, mais qu'il faut plutôt chercher activement à créer l'égalité des conditions de vie réelles ? C'est au plus tard avec la montée des mouvements ouvriers socialistes et communistes à la fin du $\mathrm{XIX}^{\mathrm{e}}$ siècle et des dictatures collectivistes au début du $X X^{\mathrm{e}}$ siècle, que le concept d'égalité prend le sens d'égalité absolue des conditions de vie - l'appréciation qui prédomine aujourd'hui encore dans l'opinion allemande, comme le révèlent les sondages.

Mais avec cette évolution, l'égalité, qui était juqu'ici la sœur de la liberté, devient son ennemie, générant dans la société un conflit de fond qui alimente jusqu'à aujourd'hui d'innombrables débats politiques : qu'est-ce qui prévaut, l'égalité, comprise comme l'objectif de créer dans la mesure du possible des conditions de vie égales, ou la liberté, entendue comme la plus grande liberté d'action individuelle possible? Aucune société ne peut échapper au choix cornélien entre ces deux visions, car elles s'excluent l'une l'autre. C'est le sociologue allemand Max Horkheimer qui, au début des années 1970, a le mieux exprimé les termes de ce conflit: "Plus il y a de justice, moins il y a de liberté; plus il y a de liberté, moins il y a de justice ». Par " justice », c'est-à-dire Gerechtigkeit en allemand, un terme qui signifie à la fois équité et justice, Horkheimer entendait l'équité sociale, une notion employée par lui, comme c'est généralement le cas, comme synonyme larvé de justice sociale. II aurait tout aussi bien pu écrire: "Plus il y a d'égalité (Gleichheit), moins il y a de liberté ; plus il y a de liberté, moins il y a d'égalité ". Tout est dit en peu de mots.

La lutte entre les valeurs que la société accorde d'une part à la liberté et, de l'autre, à l'égalité comme à la sécurité sociale et intérieure, traverse comme un fil rouge les grandes controverses politiques actuelles, par exemple les débats sur la question de savoir quelles réformes structurelles s'imposent pour sortir de la crise de la finance mondiale et de la crise de la dette souveraine. Combien d'autonomie en matière de politique économique, et donc de latitude d'action, les Etats de l'UE sont-ils prêts à abandonner pour préserver la relative égalité des conditions de vie au sein de l'UE et pour garantir la sécurité sociale dans les Etats sud-européens?

Ce schéma apparaît encore plus nettement dans les controverses de politique intérieure à propos de la politique sociale. Les réformes structurelles en la matière menées en Allemagne au début du millénaire sous l'appellation Agenda 2010 et dont on peut supposer qu'elles expliquent largement la compétitivité mondiale actuelle de l'Allemagne en comparaison de la plupart des autres Etats de l'UE, peuvent ainsi se résumer à l'abandon d'une partie, relativement petite, de la sécurité comme de l'égalité sociales, au profit d'une plus grande liberté d'action des entreprises et - corollaire par essence de la liberté -, d'une plus grande responsabilité individuelle de chaque citoyen.

Etant donné la portée économique de ce conflit foncier, il n'est pas étonnant de constater qu'il constitue l'axe principal de nombre de grandes campagnes électorales. Ce fut le cas par exemple de la campagne pour les présidentielles en France en 2012, dont l'un des pivots était la question : faut-il augmenter les impôts, c'est-à-dire réduire la liberté d'action financière d'une partie de la population, pour garantir la sécurité sociale ? Ce fut le cas également de la dernière campagne pour les présidentielles aux Etats-Unis, dominée par ce choix de priorités : faut-il une assurance maladie pour tous et financée par l'impôt ou vaut-il mieux préserver la liberté de décision individuelle ? Et point n'est besoin de beaucoup d'imagination pour penser que la campagne pour les élections au Bundestag en 2013, qui s'engage actuellement en Allemagne, sera constellée elle aussi de conflits opposant liberté et égalité ou liberté et sécurité. A preuve, le projet de l'opposition d'adopter un salaire minimum légal généralisé et des
Début du $X X^{e}$ siècle : glissement vers égalitarisme

Depuis lors,

liberté et égalité s'excluent

Le conflit entre ces deux valeurs sous-tend tous les grands débats de politique : ...

... réformes sociales...

... ou grandes campagnes électorales 
Le sens très confus de la notion d'économies budgétaires

Pour les Allemands, faire des économies $=$ réaliser des recettes

Une étude de fond sur l'approche de la liberté qu'ont les Allemands quotas féminins contraignants dans les entreprises, ou encore le débat sur les conséquences sociales de l'explosion des prix de l'électricité induite par la transition énergétique.

\section{Confusion et amalgame sont sources de problèmes de communication}

Ces considérations de principe sur le sens historique de l'hymne national allemand et les différentes interprétations du concept d'égalité ne se résument pas à un exercice purement rhétorique et dénué de toute signification pratique. Car la question de la valeur accordée par une société à la liberté est fondamentale. Et les exemples décrits montrent l'extrême importance des problèmes de communication que soulève, au sein de l'Europe et même au sein de chaque nation, la discussion sur ces concepts qui sont au fondement de nos sociétés. Or quand les concepts sont confus, propices à l'amalgame, la pensée se fait elle aussi confuse.

Le débat actuel sur la nécessité où se trouvent les Etats de l'UE de réaliser des économies budgétaires et dans quelle ampleur en est un bon exemple. Cette discussion ne peut en effet avoir de sens que si ce qu'on entend par « économies » est clairement défini. Or en Allemagne, on en est loin. Manfred Rommel, ancien maire de Stuttgart, avait eu cette formule : "faire des économies, c'est ne pas dépenser de l'argent qu'on a ", avant de poursuivre : " mais chez nous, cela veut dire ne pas dépenser de l'argent que nous n'avons pas - cela s'appelle du réalisme $»$.

Cette définition de bons sens ne s'applique dans presque aucun pays de l'UE. Au contraire même : année après année, les Etats dépensent plus de milliards qu'ils n'en perçoivent. Et pourtant, il ne se passe pas un jour sans qu'une voix s'élève dans l'opinion pour réclamer "la fin de la politique d'économies budgétaires ». Qu'entendent donc les Allemands par «faire des économies » ? C'est ce qu'a cherché à savoir l'Institut für Demoskopie d'Allensbach en procédant récemment à un sondage auprès d'un échantillon représentatif de la population. Et il est apparu que les Allemands entendent souvent par là une augmentation des recettes. Une nette majorité des sondés affirme en effet que la hausse des impôts pour les riches ou la poursuite systématique des fraudeurs fiscaux constituent des mesures appropriées d'économies budgétaires. II semble bien que la distinction entre réaliser des économies et engranger des recettes s'est diluée au point que plus aucun citoyen ne le remarque. Tout à l'inverse du particulier à qui il ne viendrait jamais à l'idée de penser qu'il réalise des économies quand il demande une hausse de son salaire à son patron. Mais dès lors qu'il s'agit du politique, la même attitude est apparamment comprise au sens de "réaliser des économies ». On est loin de la définition limpide qu'en donnait Rommel...

\section{Quel est le sens de « liberté »?}

Cet exemple montre que, avant même de se pencher sur des questions de fond relatives à la société, il faut d'abord clarifier les concepts, faute de quoi on risque de créer des malentendus. Ce n'est qu'une fois ce travail accompli qu'il est possible de comprendre les attitudes profondes ou les mentalités propres à une population.

Conjointement avec le John Stuart Mill Institut für Freiheitsforschung auprès de I'Université SRH de Heidelberg et avec I'Institut für Publizistik de I'Université de Mayence, I'Institut für Demoskopie d'Allensbach (IfD) a engagé en 2011 une étude au long cours sur la place et la valeur accordées à la liberté par la société allemande. La question de savoir quelle acception a un peuple de cette notion comme celle de la valeur accordée à celle-ci en comparaison (ou concurrence) avec d'autres aspirations comme l'égalité ou la sécurité est la clé pour mesurer 
l'adhésion d'une population à la démocratie. C'est pour cette raison qu'il convient de se livrer à une réflexion de fond sur la liberté et, avant toute chose, de bien délimiter les diverses notions et approches associées à ce concept.

Un concept communément chargé d'une connotation aussi positive que celui de liberté se trouve nécessairement au cœur de vifs conflits d'intérêts puisque, dans le débat politique, seul celui qui maîtrise les concepts est le souverain de leur interprétation. C'est ainsi qu'on peut souvent observer des tentatives de mainmise sur un contenant aussi valorisé dans le but de le charger d'un contenu qui n'a plus rien à voir avec son sens originel. Or de la sorte se multiplient les significations associées au mot "liberté ", ce qui contribue à accroître la confusion. Le discours tenu le 16 juin 2007 par Oskar Lafontaine, homme politique de gauche, en fournit un exemple symptomatique. L'orateur y expliquait que le slogan "Liberté plutôt que socialisme " (Freiheit statt Sozialismus) était un contresens et qu'il fallait lui préférer "Liberté et socialisme » ou, mieux encore : "Liberté grâce au socialisme ». Un peu plus loin, il explicitait ce qu'il entendait par là : seule une sécurité sociale généralisée permettrait au citoyen de gagner en liberté, et cette liberté ne pourrait être garantie que par le socialisme.

Cette approche place Lafontaine dans la continuité d'une longue tradition historique : voici quelque 2000 ans, déjà, la propagande de l'empereur romain Auguste avait mené à un amalgame similaire en plaçant au service du nouveau souverain la notion de " libertas " qui avait été un des concepts clés de la noble République de Rome qu'avaient anéantie de sanglantes luttes intestines et à laquelle avait succédé le régime tyrannique de l'empereur Auguste. "Libertas" renvoyait soudain à la possibilité pour chacun de ne pas être inquiété dans sa vie privée à condition de ne pas se mêler de politique et de vouer une reconnaissance absolue à l'Empereur - bref, une situation que, quelques années auparavant seulement, on aurait qualifiée d'absence de liberté. L'idée n'est pas très éloignée de remplacer la notion de liberté de décision et d'action de chaque citoyen par la promesse d'une sécurité sociale et de justifier ce glissement de sens par la notion de liberté.

Le grand dictionnaire de la langue allemande ("Deutsches Wörterbuch ») des Grimm, publié en 1878, mais qui fait toujours référence, contient dix définitions différentes du concept de liberté. Aucune nuance, aussi ténue soit-elle, n'est oubliée. Mais jamais, malgré leur désir d'exhaustivité, l'idée n'est venue aux Frères Grimm de comprendre la liberté comme la conséquence d'une aide sociale nourricière que seul, de surcroît, permettrait de garantir le socialisme, c'est-à-dire un système de société qui ne peut s'imposer que par la contrainte et une profonde ingérence dans l'organisation de la vie des citoyens.

On comprend mieux la confusion du sens de liberté, la variété des malentendus possibles, quand on étudie la valeur accordée à ce concept. Or le flou conceptuel est tel dans les débats politiques et intellectuels qu'on ne peut guère s'attendre à ce que la population en ait une conception plus claire. Ce ressenti et ces appréciations indéterminées, souvent même contradictoires, qui sont les caractéristiques de l'opinion publique, ne peuvent être condamnés. Ils ne peuvent pas non plus être modifiés consciemment. Ils font tout simplement partie intégrante de cette réalité qu'il s'agit de décrire.

Par ailleurs, avant de se pencher plus avant sur le thème de la liberté dans le cadre d'un sondage, il faut choisir les aspects qui ne seront évoqués qu'à la marge ou pas du tout. Dans le sondage représentatif de l'IfD présenté cidessous, la liberté politique n'a par exemple été considérée qu'à la marge. Assurément, dans une démocratie, la liberté dont dispose le citoyen de participer à la vie politique, son droit à s'informer librement, à exprimer son opinion sans être inquiété par les pouvoirs publics, à voter et à se présenter aux élections, sont des éléments d'une importance incontestable. Mais il s'agit là de principes fondamentaux de droit constitutionnel qui ne sont guère mis en doute dans la République fédérale d'Allemagne.
Liberté plutôt que socialisme?

Liberté $=$ promesse de sécurité sociale?

Le flou conceptuel entraîne des visions contradictoires

Le sondage de I'IfD ne traite pas de la liberté politique ... 
... ni de la liberté économique...

... ni de la liberté de pensée au sens de Luther

Le sondage porte sur la liberté en tant que valeur fondatrice de la société

Il distingue 3 approches : liberté $=$ pas de misère sociale,..

... liberté = libertinage, $\ldots$

... liberté $=$ auto-détermination
Dans ce sondage, la liberté économique n'est pas placée au centre de l'intérêt, aussi importante soit-elle pour le bien-être d'une société. Elle y est certes considérée, en tant qu'un aspect subsidiaire de la thématique, le seul toutefois auquel le débat public accorde de temps en temps quelque attention. Les grands indices internationaux de liberté que publie l'Institut Fraser au Canada ou la Heritage Foundation aux Etats-Unis se concentrent ainsi exclusivement sur ce point. Mais la question de savoir à quel degré une société est attachée à la valeur liberté dépasse de loin la problématique économique. Elle concerne en effet le domaine des droits du citoyen, la possibilité pour lui de faire librement des choix de vie sans qu'ils soient trop entravés par l'Etat et sa réglementation, sa disposition à se prendre en charge lui-même et à assumer ses responsablités, ou sa capacité à faire preuve de tolérance vis-à-vis d'opinions ou de projets de vie alternatifs, pour ne citer que quelques exemples.

Un autre aspect intéressant de la thématique de la liberté a dû être écarté dans ce sondage : l'idée de l'autonomie intellectuelle, d'une pensée indépendante ne cédant pas aux courants du Zeitgeist ou aux impératifs de l'ordre séculier. La doctrine protestante de la liberté telle que l'avait développée Luther dans son Traité "De libertate christiana » (Freiheit des Christenmenschen) et selon laquelle l'Homme ne doit de comptes qu'à Dieu et en tire une liberté individuelle, personnelle, indépendante de la liberté politique ou de celle de la société, est certes d'importance fondamentale en matière d'histoire des religions ou des civilisations. Mais elle n'est d'aucun secours pour savoir quel rôle devrait jouer la valeur accordée à la liberté dans l'organisation d'une société moderne.

Mais même lorsqu'on se contente de concentrer l'analyse sur la liberté en tant que valeur fondatrice de la société, il faut encore distinguer entre trois approches qui entrent partiellement en contradiction.

Il s'agit d'une part de la notion de liberté telle qu'elle est typiquement propagée dans le contexte de l'idéal des sociétés collectivistes, et telle qu'elle perce dans le discours d'Oskar Lafontaine évoqué ci-dessus. La liberté y est comprise avant tout comme le fait d'être libre de misère sociale, c'est-à-dire comme quelque chose qui est accordé au citoyen par un Etat puissant et prévoyant, éventuellement même au prix de l'absence de liberté politique et d'une profonde ingérence de l'Etat dans la liberté de décision et d'action de chacun. Comme esquissé plus haut, une telle vision n'est guère compatible avec la signification traditionnellement accordée au mot liberté; elle est même en totale contradiction avec l'idée de la liberté d'action de l'individu. II reste toutefois à déterminer quel rôle joue cette approche dans l'opinion et combien de citoyens pensent en premier lieu à la protection sociale quand ils entendent le mot liberté.

La deuxième approche de la notion de liberté peut se résumer par le terme de "libertinage », c'est-à-dire la possibilité de faire (ou de ne pas faire) ce qu'il vous plaît, de n'être tenu par aucune règle ou norme et, cas extrême, de ne pas avoir à tenir compte des autres ni à supporter les conséquences de ses actes, donc à en porter la responsabilité.

Dans la troisième approche, liberté s'entend comme la possibilité de chacun à l'auto-détermination, la possibilité de tout faire pour réussir dans la vie, de saisir les opportunités et d'assumer pleinement la responsabilité de tout ce qu'implique le choix de vie. Dans cette acception, la liberté n'apporte pas seulement des opportunités, mais elle exige aussi de chacun qu'il soit actif et qu'il s'efforce. C'est cette approche de la liberté qui, lorsqu'elle est considérée comme un principe fondateur de la société, entre en permanence en conflit avec les objectifs d'égalité et de sécurité. C'est elle aussi qui sous-tend souvent de manière plus ou moins explicite les lignes argumentaires dans les débats sur l'économie ou les questions de société (à moins que, comme dans l'exemple cité plus haut, il ne s'agisse d'une de ces tentatives d'appropriation, voire de confiscation du concept). 
Mais cette vision d'une liberté basée sur son exercice actif, donc la liberté d'agir et de faire des choix, est-elle prédominante dans l'opinion ? Pour permettre aux sondés de cette enquête représentative de répondre à cette question, les trois principales approches du concept de liberté ont été décrites avec des mots très simples sur des fiches présentées aux personnes interrogées. La présentation était suivie de la question : "Quel sens accordez-vous à liberté ? Pouvez-vous me dire laquelle de ces trois fiches exprime le plus fidèlement ce que vous pensez ? ». $24 \%$ des sondés ont répondu : "Liberté signifie être libéré de misère sociale, de pauvreté, d'absence de domicile fixe et de chômage "; 21 \% d'entre eux ont affirmé : "Liberté signifie pouvoir faire ce qu'il vous plaît, partir en voyage où on veut, vivre comme on veut ». Une nette majorité de $52 \%$ a opté pour: "Liberté signifie être autonome et assumer ses responsabilités, choisir librement d'exercer un métier plutôt qu'un autre, de vivre dans le pays ou la ville qui vous plaît, et pouvoir s'engager pour un objectif qu'on souhaite atteindre ".

Malgré toute la confusion constatée dans le débat public quant au sens à accorder au concept de liberté, les Allemands partagent donc largement la troisième approche, celle de la liberté de décision et d'action (voir tableau 1). Et on peut supposer en conséquence que, à chaque fois que la question de la liberté est évoquée dans un sondage, les réponses sont déterminées par cette lecture du concept.
Les Allemands partagent largement l'approche de la liberté responsable

Tableau 1

Que signifie liberté ?

\begin{tabular}{|c|c|c|c|}
\hline \multicolumn{4}{|c|}{$\begin{array}{l}\text { Question : "Quel sens accordez-vous à liberté ? Pouvez-vous me dire laquelle de ces trois fiches } \\
\text { exprime le plus fidèlement ce que vous pensez? " (présentation de trois fiches de réponses). }\end{array}$} \\
\hline \multicolumn{4}{|c|}{ RFA, population des 16 ans et plus, réponses en $\%$} \\
\hline & RFA & ouest & est \\
\hline $\begin{array}{l}\text { "Liberté signifie être libéré de misère sociale, de pauvreté, } \\
\text { d'absence de domicile fixe et de chômage " }\end{array}$ & 24 & 21 & 36 \\
\hline $\begin{array}{l}\text { "Liberté signifie pouvoir faire ce qu'il vous plaît, partir en } \\
\text { voyage où on veut, vivre comme on veut " }\end{array}$ & 21 & 21 & 20 \\
\hline $\begin{array}{l}\text { "Liberté signifie être autonome et assumer ses responsabilités, } \\
\text { choisir librement d'exercer un métier plutôt qu'un autre, de } \\
\text { vivre dans le pays ou la ville qui vous plaît, et pouvoir } \\
\text { s'engager pour un objectif qu'on souhaite atteindre" }\end{array}$ & 52 & 55 & 42 \\
\hline n.s.p. & 3 & 3 & 2 \\
\hline Total & 100 & 100 & 100 \\
\hline n (nombre de personnes interrogées) & 1792 & 1183 & 609 \\
\hline
\end{tabular}

Source : Allensbacher Archiv, sondage IfD-Umfrage n 10077, août 2011.

\section{La valeur accordée à la liberté}

Le conflit foncier qui oppose les principes de liberté et d'égalité préoccupe les chercheurs de l'IfD depuis des décennies ; il est régulièrement abordé dans ses sondages représentatifs. II se présente sous forme de 'questions-dialogues' dont la formulation peut varier : on présente au sondé une image montrant deux personnages esquissés sous forme de silhouettes; comme dans une bande dessinée, ce qu'ils disent figure dans une bulle. L'un des protagonistes dit : " Je trouve certes que la liberté et la plus grande égalité (ou justice sociale) possible, sont aussi importantes l'une que l'autre, mais si j'avais à choisir, je dirais que le plus important pour moi, c'est la liberté individuelle, c'est-à-dire que chacun peut vivre en liberté et s'épanouir librement ". L'autre dit au contraire: "Bien sûr, la liberté et l'égalité ou la justice sociale sont importantes, mais si j'avais à choisir entre les deux, le plus important pour moi, ce serait la plus grande égalité possible, c'est-à-dire quand personne n'est défavorisé et quand les différences sociales ne sont pas si grandes". Suit alors la question: "Voici deux personnes qui discutent pour savoir ce qui est le plus important : la liberté
L'IfD se penche depuis 15 ans sur le conflit liberté vs égalité 
ou une plus grande égalité ou justice sociale possible. Lisez ce qu'elles disent. Laquelle des deux exprime plutôt ce que vous pensez vous aussi ? ".

\section{Graphique 1}

\section{Liberté et égalité (ensemble de la RFA)}

Question : "Voici deux personnes qui discutent pour savoir ce qui est le plus important : la liberté ou une plus grande égalité (ou justice sociale) possible. Lisez ce qu'elles disent. Laquelle des deux exprime plutôt ce que vous pensez vous aussi ? » (présentation de l'image).

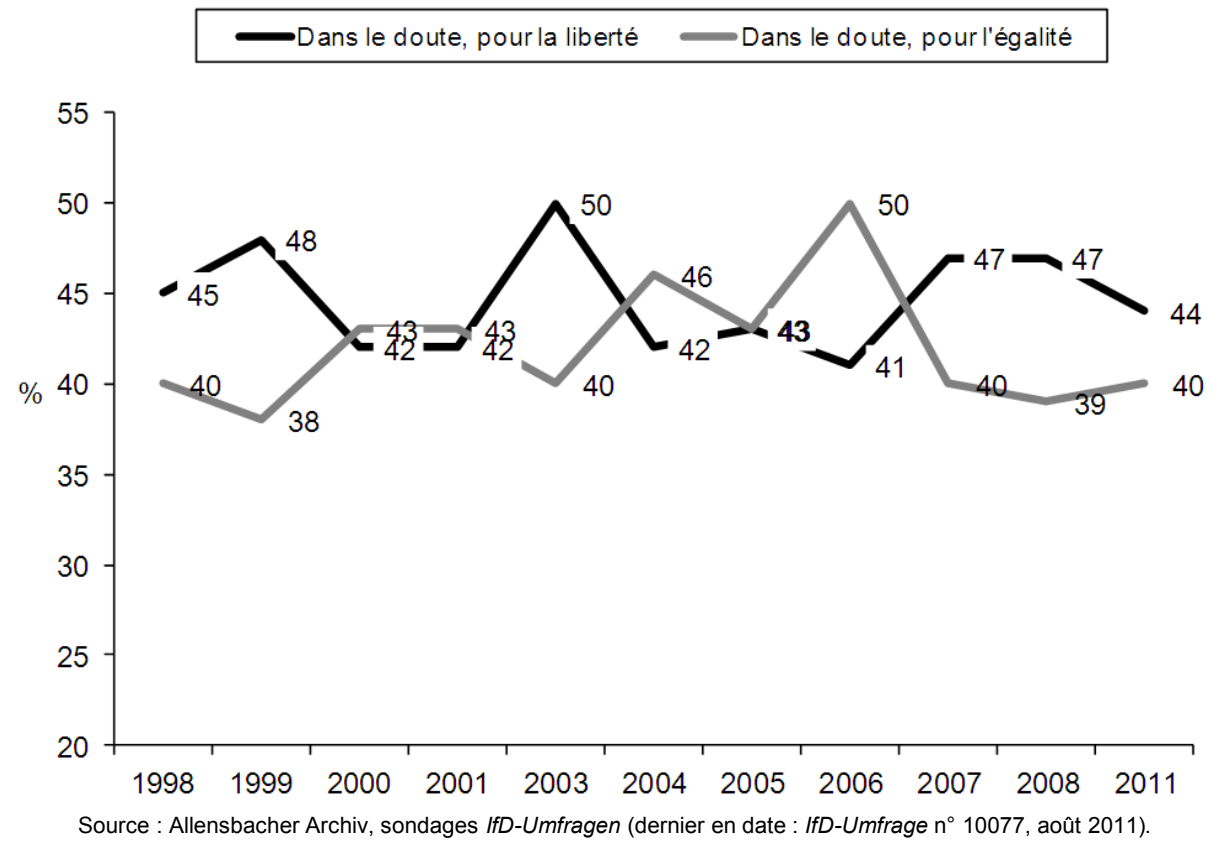

Les Allemands accordent plus d'importance à la liberté que les Français
Depuis près de quinze ans, la part de ceux qui préfèrent la liberté et de ceux qui préfèrent l'égalité est restée quasi identique, comme le montre le graphique 1. Le politologue américain Samuel Huntington avait un jour expliqué que l'évolution démocratique d'une société dépend, au fond, de la priorité qu'elle accorde toujours, en cas de doute, à la valeur liberté. Actuellement, la société allemande en est loin. Mais elle n'est pas la seule en Europe, comme le révèlent les conclusions d'une étude comparée internationale sur les valeurs menée en 2005 (voir tableau 2). A l'époque de ce sondage en tout cas, le degré d'importance que les Allemands accordaient à la liberté était comparable à celui des Britanniques et des Polonais. Les Français, par contre, lui accordaient nettement moins d'importance.

Tableau 2

Qu'est-ce qui est prioritaire : la liberté ou l'égalité ? Comparaison européenne, 2005

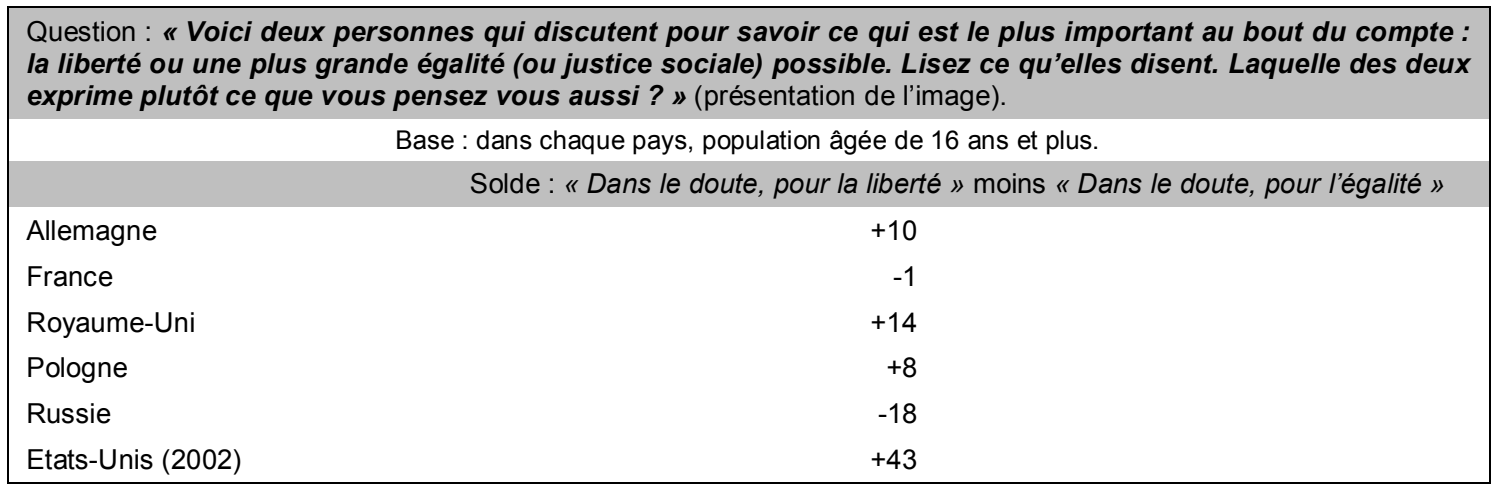

Source : Allensbacher Archiv, sondage IfD-Umfrage $n^{\circ}$ 7049, et sondage IMAS-Umfrage n 5029. 


\section{"Chacun est l'artisan de son propre bonheur »}

La valeur accordée à la liberté par les Allemands n'en a pas moins évolué au cours des dernières années. Quand on quitte en effet le terrain de l'abstraction qu'est la problématique de la hiérarchie entre liberté et égalité et qu'on traduit ces deux concepts à l'aide de notions plus proches de la vie quotidienne, le tableau change alors du tout au tout. Depuis 1955, une des questions que pose régulièrement l'IfD est la suivante : "Deux hommes parlent de la vie. L'un dit : 'chacun est l'artisan de son propre bonheur. Quelqu'un qui veut bien se donner du mal réussira dans la vie'. L'autre dit : La réalité, c'est que certains sont en haut de l'échelle, d'autres en bas et que, dans les circonstances actuelles, ceux-là ne parviendront jamais à grimper plus haut malgré tout le mal qu'ils se donnent'. Quel est votre avis à vous : qui des deux a plutôt raison?"

L'importance de cette question tient à ce qu'elle cherche à capter la vision de l'Homme sous-jacente à la valeur fondamentale accordée à la liberté. Seuls ceux qui pensent que la plupart des individus sont prêts à et capables de prendre eux-mêmes leur sort en main approuveront la liberté comme le principe selon lequel doit être organisée la société. Ceux qui pensent, à l'inverse, que les individus sont livrés pieds et poings liés au système n'accorderont qu'une faible importance à la liberté d'action, ce qui les amène à plaider pour un maximum de prévoyance et de redistribution matérielle de la part de l'Etat.

\section{Grahique 2}

\section{"Chacun est l'artisan de son propre bonheur »}

Question : "Deux hommes parlent de la vie (...) Quel est votre avis à vous : qui des deux a plutôt raison?"
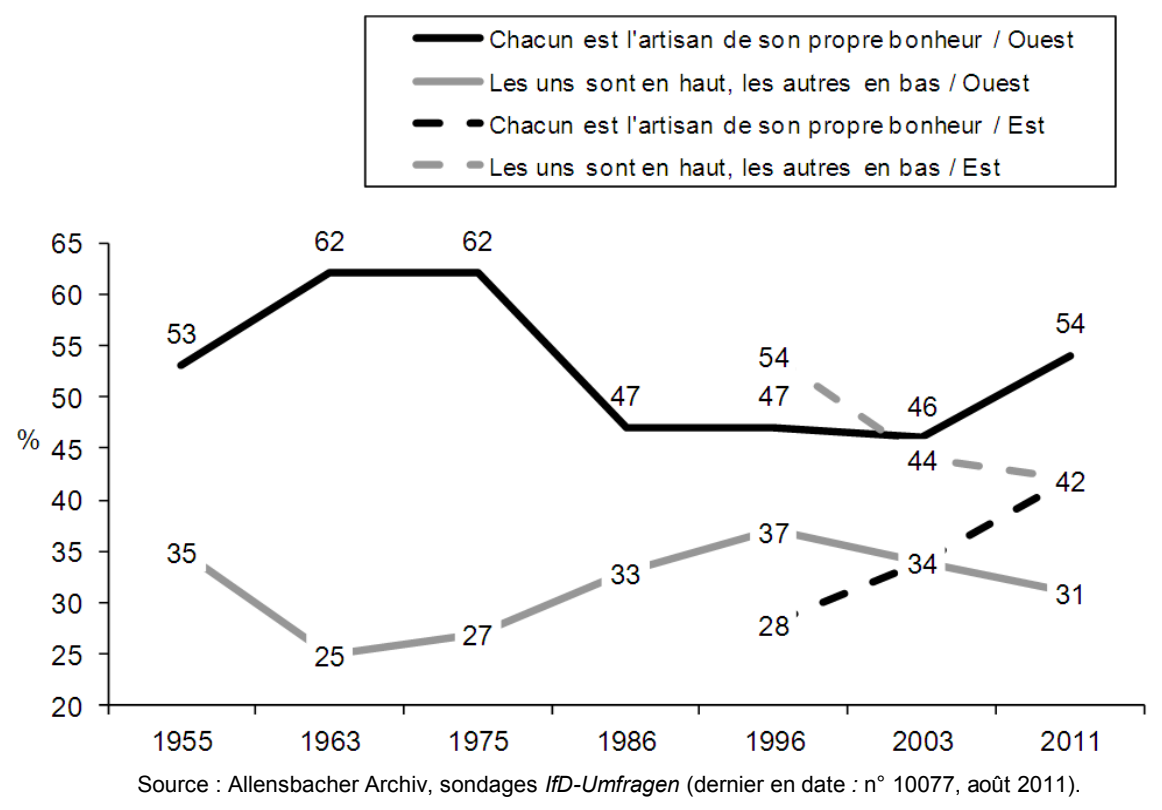

Le graphique 2 retrace l'évolution des réponses à cette question en distinguant entre celles des Allemands de l'est et de l'ouest. On voit s'y profiler le même cheminement que pour la question sur la priorité entre liberté et égalité : en Allemagne de l'ouest, une nette majorité des sondés a toujours affirmé que chacun est l'artisan de son propre bonheur, cette majorité étant particulièrement prononcée dans les années 1950, 1960 et au début des années 1970. Dans les décennies suivantes, elle est tombée à un niveau légèrement inférieur, probablement sous l'effet de la mutation des valeurs qui avait fait reculer dans l'opinion l'adhésion aux valeurs « bourgeoises » traditionnelles [bürgerlich : c'est-àdire les vertus de la citoyenneté et de la classe moyenne au fondement de la 'société civile' allemande ; voir REA 84/2007 ; NdT]. Le sondage réalisé en août
Mais qu'entendent concrètement les Allemands par liberté ?
Allemands de l'ouest et de l'est se rejoignent aujourd'hui 
Des différences d'approche entre générations

"Chacun est l'artisan de son propre bonheur" gagne du terrain chez les jeunes à l'ouest...
2011 montre ensuite un retour au niveau des années 1950. Dans les nouveaux Länder (ex-RDA), l'évolution est différente : en 1996, année où la question y fut posée pour la première fois, une nette majorité de $54 \%$ des personnes interrogées avaient répondu "certains sont en haut, d'autres en bas ». On pouvait s'attendre à un tel taux de la part d'une population dont la vision du monde avait été forgée par un régime socialiste (voir REA 98-99/2010). Mais ensuite, au fil des ans, l'opinion des Allemands de l'est s'est modifiée nettement. Et aujourd'hui, $42 \%$ d'entre eux seulement partagent encore cette assertion, la même proportion pensant désormais que chacun est l'artisan de son bonheur.

Tout porte à croire que ce regain d'adhésion à la valeur accordée à la liberté n'a rien d'un pénomène de mode éphémère, mais qu'il s'agit bien plutôt d'un profond revirement de tendance. C'est ce que révèle la comparaison de l'évolution dans le temps des réponses des différentes générations. Depuis le début de cette enquête (1955), la part des Allemands de l'ouest affirmant que chacun est l'artisan de son propre bonheur est inférieure chez les moins de 30 ans que chez les trentenaires ou les plus âgés. L'écart était faible durant les années 1950 et 1960, mais s'est nettement creusé ensuite avant d'atteindre son maximum dans les années 1980.

Les différences constatées entre les générations en matière de valeurs sont toujours le signe qu'une société est en train de profondément changer ; c'est la jeune génération qui montre la voie que va suivre à terme la société dans son entier puisque c'est la vision du monde des plus jeunes qui finit par s'imposer au fil des ans. Et en toute conséquence, à partir des années 1970 a commencé à baisser dans l'opinion la part de ceux pensant que la plupart des gens peuvent réussir dans la vie s'ils se donnent du mal. Mais, bien que l'écart entre les générations se soit réduit, le schéma qui prévalait au cours des décennies écoulées s'est maintenu jusqu'en 2003. Ce n'est qu'ensuite que l'opinion a profondément changé : en 2011, non seulement, l'avis que " chacun est l'artisan de son propre bonheur » a atteint dans l'ensemble de l'opinion un niveau jamais enregistré depuis les années 1970, mais pour la première fois aussi, cette approche était nettement plus partagée par les moins de 30 ans que les plus âgés (voir graphique 3). C'est là indubitablement le signe que cette opinion va gagner du terrain au cours des années à venir.

Graphique 3

"II y a à nouveau de plus en plus de jeunes qui disent : 'chacun est l'artisan de son propre bonheur' » - Allemagne de l'ouest

Question : "Deux hommes parlent de la vie (...) Quel est votre avis à vous : qui des deux a plutôt raison? 》

Réponses : "Chacun est l'artisan de son propre bonheur"

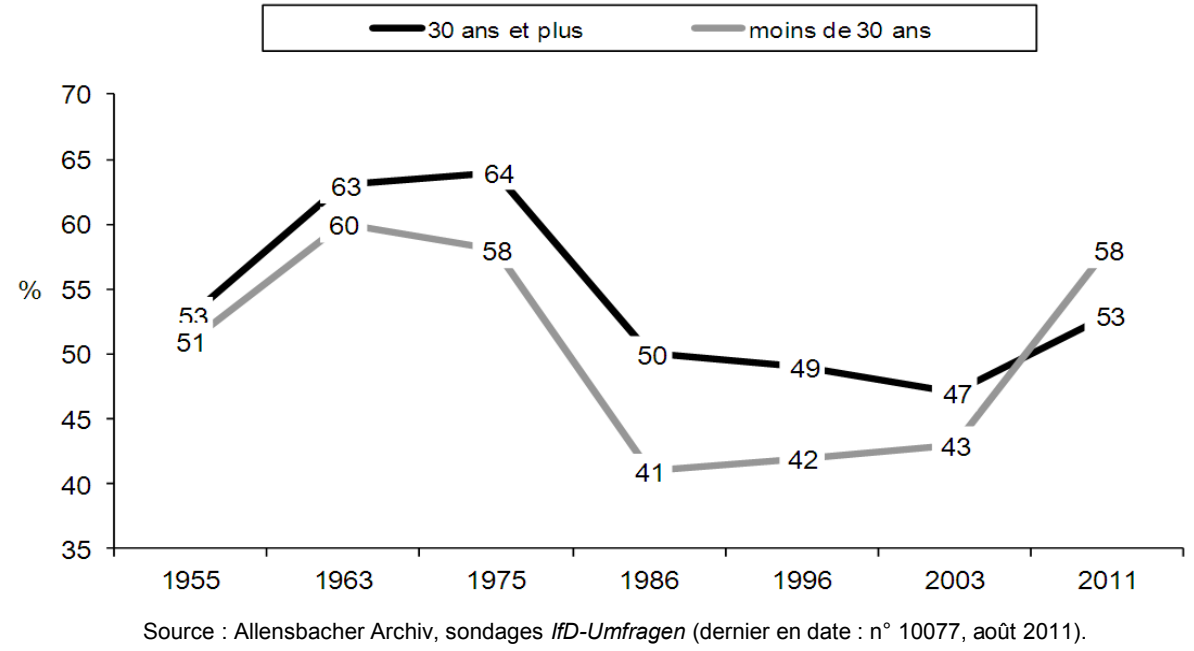


L'évolution observée dans les nouveaux Länder (issus de la RDA) mérite une attention particulière. Une courte majorité de $46 \%$ des moins de 30 ans comme des plus âgés y pensent que le sort de chacun est déterminé par le système, et seulement 39 \% que chacun est l'artisan de son propre bonheur. On retrouve ici les différences classiques observées dans la vision du monde qu'ont les Allemands de l'ouest et de l'est du fait de leur histoire. Or les réponses des moins de 30 ans sont tout autres. A l'ouest, celles des jeunes se distinguent à peine de celles des plus âgés. A l'est en revanche, les moins de 30 ans sont nettement plus nombreux que leurs parents ou grand-parents à affirmer leur attachement à la liberté : $56 \%$ d'entre eux, soit à peu près autant qu'à l'ouest, pensent que chacun est l'artisan de son propre bonheur (voir tableau 3). Ce taux de réponses peut en surprendre plus d'un, puisqu'il est en franche contradiction avec le stéréotype, très répandu il y a quelques années, d'une jeunesse est-allemande dépossédée de tout espoir en l'avenir. Et on imagine d'autant plus aisément combien cette approche des jeunes Allemands de l'est peut être source de conflit au sein des familles quand parents et enfants s'opposent aussi franchement sur une valeur fondamentale.

Tableau 3

La valeur accordée à la liberté. Un conflit générationnel en Allemagne de l'est

\begin{tabular}{|c|c|c|c|c|}
\hline \multicolumn{5}{|c|}{ Question : « Deux hommes parlent de la vie (...) Quel est votre avis à vous : qui des deux a plutôt raison ?» } \\
\hline \multirow{2}{*}{$\begin{array}{l}\text { RFA } \\
\text { Personnes interrogées de } \ldots\end{array}$} & \multicolumn{2}{|c|}{ ouest } & \multicolumn{2}{|c|}{ est } \\
\hline & $\begin{array}{l}\text { moins de } \\
30 \text { ans }\end{array}$ & $\begin{array}{l}30 \text { ans et } \\
\text { plus }\end{array}$ & $\begin{array}{l}\text { moins de } \\
30 \text { ans }\end{array}$ & $\begin{array}{l}30 \text { ans } \\
\text { et plus }\end{array}$ \\
\hline \multicolumn{5}{|l|}{ Réponses en \% } \\
\hline $\begin{array}{l}\text { "Chacun est l'artisan de son propre bonheur. Quelqu'un } \\
\text { qui veut bien se donner du mal réussira dans la vie" }\end{array}$ & 58 & 53 & 56 & 39 \\
\hline $\begin{array}{l}\text { "La réalité, c'est que certains sont en haut de l'échelle, } \\
\text { d'autres en bas et que, dans les circonstances actuelles, } \\
\text { ceux-là ne parviendront jamais à grimper plus haut malgré } \\
\text { tout le mal qu'ils se donnent " }\end{array}$ & 26 & 32 & 26 & 46 \\
\hline n.s.p. & 16 & 15 & 18 & 15 \\
\hline Total & 100 & 100 & 100 & 100 \\
\hline n (nombre de personnes interrogées) & 218 & 965 & 109 & 500 \\
\hline
\end{tabular}

Source : Allensbacher Archiv, sondage IfD-Umfrage n 10077, août 2011.

\section{La peur de la liberté}

On vient de le voir, la part de ceux qui pensent que, généralement, chacun est maître de son destin augmente parmi les Allemands. En toute logique, cette approche constitue la base de l'adhésion croissante à la valeur liberté. On pourrait dès lors penser que dans une société ainsi configurée, la méfiance envers l'ingérence de l'Etat dans la vie privée des citoyens devrait augmenter en parallèle, puisqu'une vision du monde axée sur la liberté de l'individu ou au contraire sur la prévoyance de l'Etat s'excluent largement l'une l'autre. Elles expriment en effet deux objectifs sociétaux contradictoires: d'une part, celui d'une liberté d'action de l'individu qui soit la plus grande possible et, d'autre part, celui de son besoin de règles lui apportant prévoyance et sécurité. Ou pour le dire autrement : il s'agit du conflit entre les deux objectifs que sont d'une part l'autoorganisation, et d'autre part le pilotage d'une société par l'Etat.

Or en Allemagne, l'approche de l'appel à l'Etat et sa prévoyance n'a pas reculé au cours des dernières années, mais bien au contraire augmenté. C'est tout particulièrement vrai de l'appel à l'intervention de l'Etat dans le système économique - et ce, depuis bien avant qu'éclate la crise de la finance mondiale. Les réponses apportées à une 'question-dialogue' sur ce que les Allemands pensent de l'économie de marché sont symptomatiques à cet égard. Selon le principe décrit ci-dessus, les personnes interrogées ont à choisir entre deux ar-

Economie libérale et justice sociale? 
$44 \%$ pensent aujourd'hui que l'économie de marché génère l'injustice

La liberté de plus en plus ressentie comme une menace, ...

... surtout en cas de conflit guments avancés par deux silhouettes sur une image. Le premier argument est le suivant: "Seule une économie libérale [freie Marktwirtschaft : littéralement « économie de marché libre »; NdT] permet d'assurer une sécurité sociale. Un Etat a besoin de beaucoup d'argent pour soutenir les pauvres et les faibles dans la société, et cet argent, il ne l'a à sa disposition que dans une économie de marché qui fonctionne correctement ". L'argument opposé est : "Je ne partage pas cet avis. Une économie libérale mène automatiquement à l'injustice sociale. Les riches s'enrichissent toujours plus, et les pauvres ne cessent de s'apauvrir ».

En 2004 encore, une majorité relative de $48 \%$ approuvait la première assertion, défendant ainsi le principe de l'économie de marché, seuls $34 \%$ estimant que cette dernière mène automatiquement à l'injustice sociale. Huit ans plus tard, le tableau est très différent : en août 2011, une courte majorité relative de $44 \%$ pense que l'économie de marché mène automatiquement à l'injustice sociale, et seulement $39 \%$ sont d'avis contraire.

Il est riche d'enseignements de regarder de plus près les visions du monde qui se cachent derrière ce refus croissant de l'économie de marché. On découvre ainsi que la liberté est ressentie littéralement comme une menace par de nombreux Allemands. C'est ce que révèlent les réponses à une question demandant aux sondés d'imaginer deux Etats : le premier, " un Etat qui se préoccupe beaucoup de ses citoyens, leur apporte beaucoup de sécurité/prévoyance et qui intervient dans l'économie dès que surviennent des problèmes "; l'autre, " un Etat qui n'intervient pratiquement pas dans l'économie et qui se contente d'assurer les domaines les plus indispensables en matière de sécurité/prévoyance ». Ensuite, on demande aux personnes interrogées de dire dans lequel des deux pays ils pensent qu'il y a le plus de justice, le plus de prospérité, le plus de liberté, et lequel est le plus humain.

Les réponses (voir tableau 4) montrent que, certes, les Allemands concèdent volontiers que dans un Etat libéral qui n'intervient pas dans l'économie, il y a une plus grande liberté que dans un Etat social qui joue un rôle actif dans l'économie. Mais ce dernier est ressenti comme plus juste. Par ailleurs, une nette majorité d'Allemands pense que l'Etat qui intervient jusque dans la vie des citoyens apporte une plus grande prospérité. Plus important : l'Etat prévoyance est ressenti comme plus humain que l'Etat libéral.

Tableau 4

Deux Etats : liberté s'oppose à humanité

\begin{tabular}{|lcc|}
\hline $\begin{array}{l}\text { Question : « Si vous aviez à comparer deux Etats (...) } \\
\text { Réponses (en \%) : }\end{array}$ & $\begin{array}{c}\text { Etat } \\
\text { protecteur/prévoyant }\end{array}$ & $\begin{array}{c}\text { Etat } \\
\text { libéral }\end{array}$ \\
\hline " A votre avis, dans lequel des deux y a-t-il plus de justice ? » & 55 & 21 \\
" A votre avis, dans lequel des deux Etats y a-t-il une plus & 45 & 28 \\
grande prospérité ? » & & 16 \\
" A votre avis, lequel des deux Etats est le plus humain ? » & 64 & 53 \\
" Dans lequel de ces deux Etats la liberté est-elle la plus & 23 & \\
grande ? " & & \\
\hline
\end{tabular}

Source : Allensbacher Archiv, sondage IfD-Umfrage $\mathrm{n}^{\circ}$ 10077, août 2011.

Les réponses des sondés révèlent donc vraisemblablement l'existence dans leur subconscient d'une attitude qui s'oppose en Allemagne à une plus large adhésion aux principes fondés sur la liberté; personne ou presque ne répondrait oui si on soumettait aux Allemands une question directe décrivant la liberté comme inhumaine. Or c'est cette même approche qui transparaît dans les réponses à la question sur les deux Etats : la liberté s'y oppose au caractère humain. Autrement dit: la liberté suscite une large adhésion lorsque les questions sont formulées de manière générale; mais en situation de conflit, c'est la peur de la liberté qui prédomine. 


\section{SOCIÉTÉ}

\section{La demande d'interdits}

II est donc parfaitement logique que l'opinion demande à l'Etat de plus en plus de contrôles et d'interdits dans les domaines les plus divers. Une question posée aux sondés leur demande quelles choses et quels comportements devraient à leur avis être interdits par l'Etat. On leur présente à cet effet une liste de 21 éléments dont certains sont effectivement frappés d'interdit, mais dans la plupart des cas, il s'agit de choses ou comportements qui ne sont aucunement interdits ou qui sont autorisés sous certaines conditions.

Cette question a été posée pour la première fois en 2003. A l'époque, les personnes interrogées s'étaient majoritairement prononcées pour interdire les films, vidéos et jeux électroniques contenant de nombreuses scènes de violence, les partis de l'extrême droite et les aliments dangereux pour la santé.

\section{Graphique 4}

\section{La demande d'interdits}

Question: "Que cela soit effectivement interdit ou non : à votre avis, qu'est-ce que l'Etat devrait interdire dans tous les cas, dans quels domaines l'Etat doit-il protéger les gens d'eux-mêmes ? Sur cette liste, quels sont les points que vous nommeriez? » (présentation de la liste).

Extrait des réponses :

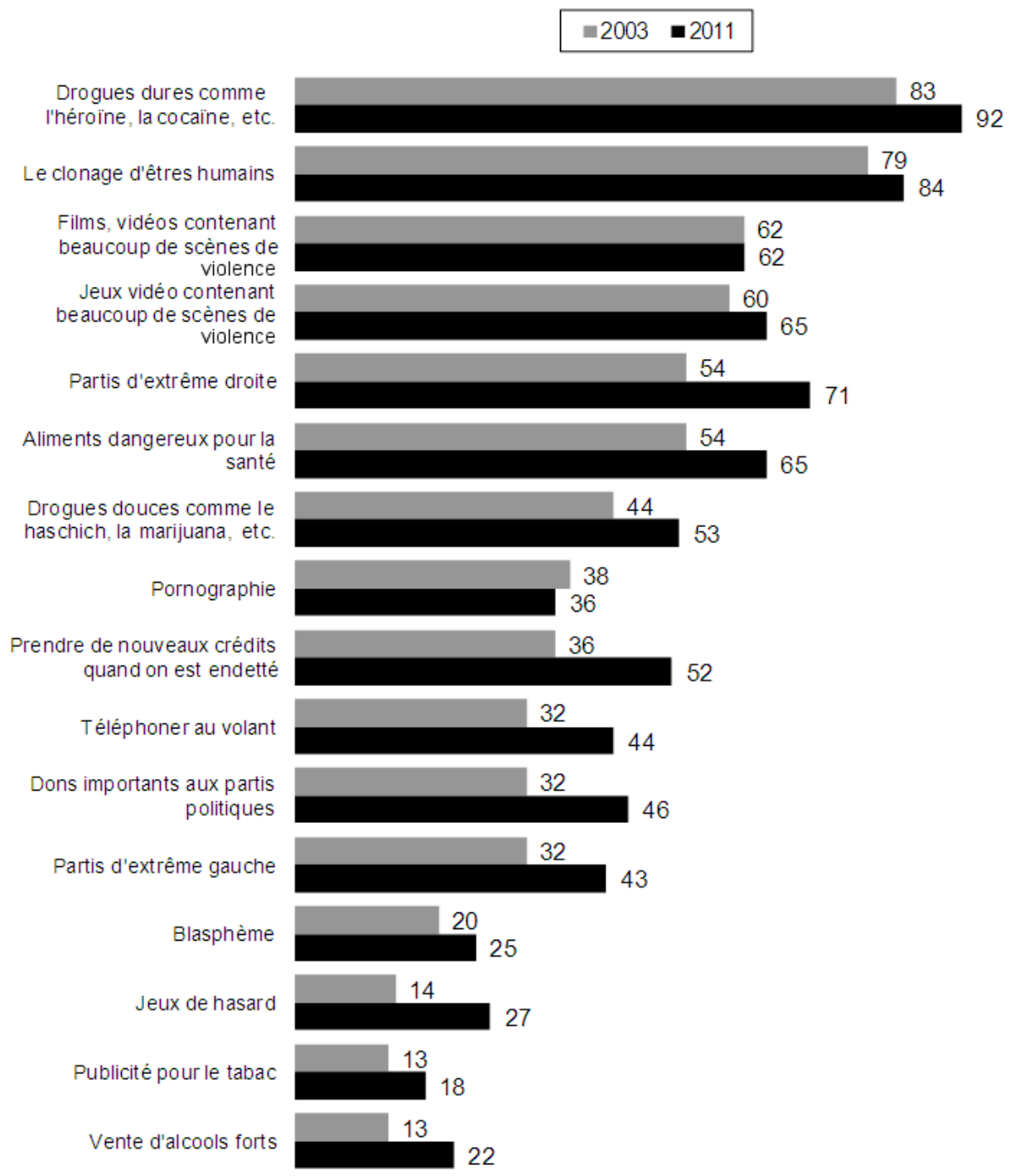


.. la demande d'interdits a explosé

Les Allemands ne perçoivent plus guère la contradiction entre interdit et liberté

La pression sociale est déterminante

C'est par elle que le libéralisme favorise la cohésion sociale
Quand la question fut reposée en 2011, il s'est révélé que le nombre de demande d'interdits avait massivement augmenté pour presque tous les points de la liste. Alors qu'en 2003, les Allemands étaient $54 \%$ à réclamer l'interdiction d'aliments dangereux pour la santé, ils étaient $65 \%$ à le faire en 2011. Les drogues douces comme le haschich ou la marijuana devaient être frappées d'interdit pour $44 \%$ des sondés en 2003, pour $53 \%$ en 2011. En 2003, $36 \%$ plaidaient pour interdire aux personnes endettées l'accès au crédit, ils étaient $52 \%$ en 2011. Qu'il s'agisse de partis extrémistes, de dons importants aux partis politiques, de jeux de hasard, de publicité pour le tabac, de grosses cylindrées ou d'alcool fort - la demande d'interdits a augmenté tous azimuts. Sur aucun point de la liste, le nombre de ceux plaidant pour l'interdit n'a reculé significativement (voir graphique 4). La même tendance s'observe aussi dans les réponses à une autre question où on demandait aux personnes interrogées de dire quels domaines ils désiraient voir contrôlés de manière conséquente par l'Etat : le désir d'un contrôle par l'Etat a considérablement augmenté dans presque tous les domaines.

Les sondages montrent donc des opinions en apparence contradictoires. D'un côté, le climat de l'opinion tend à évoluer vers une plus large adhésion à la valeur liberté ; de l'autre, la demande faite à l'Etat d'intervenir dans la vie des citoyens augmente, ce qui revient finalement à revendiquer que soit restreinte la liberté individuelle. Fait révélateur, ces demandes sont formulées par les personnes interrogées sans aucun lien avec leur opinion foncière sur la valeur à accorder à la liberté. En effet, ceux qui préfèrent la liberté à l'égalité approuvent pareillement la création de nouveaux interdits que ceux qui donnent priorité à l'égalité. Ce constat étonnant montre que nombreux sont les Allemands qui ne perçoivent pas la nature du lien entre la liberté individuelle et l'extension du contrôle exercé par l'Etat sur leur propre vie. A l'évidence, la revendication de la liberté et la demande d'interdits ne sont pas perçues comme contradictoires.

\section{Perception subjective de la liberté et degré de tolérance dans la société}

Un autre aspect de la thématique de la liberté est la question de savoir à quel degré la société allemande allemande se montre ouverte et tolérante vis-à-vis des opinions divergentes et celle de savoir à quel degré les Allemands se sentent libres dans leur vie personnelle.

Le fait qu'une société est libérale n'implique pas nécessairement que, dans leur vie quotidienne, les citoyens s'y sentent libres effectivement. II n'y a en effet pas de lien direct entre le libéralisme d'une société et la perception individuelle de la liberté ; celui-ci est au contraire d'une grande complexité. Déjà, lors de son voyage aux Etats-Unis en 1831, Alexis de Tocqueville avait constaté avec stupéfaction que ses citoyens, alors même qu'ils s'étaient dotés de la Constitution la plus libérale de l'époque, vivaient dans un climat social où la liberté individuelle était absente. Cette non-liberté qu'évoquait Tocqueville n'émanait pas des institutions ou des autorités du pays, mais émanait au contraire des citoyens eux-mêmes. C'étaient eux-mêmes qui s'étaient donné une organisation faite de "lois non écrites », autant de normes ou conventions qu'il n'était pas possible d'enfreindre sans s'attirer la réprobation des concitoyens. Aujourd'hui, on parlerait de "politiquement correct ». Ces mécanismes de la formation de l'opinion publique sont bien connus; Elisabeth Noelle-Neumann les a subsumés dans sa théorie de la « spirale du silence » (voir REA 84/2007).

L'articulation entre la liberté politique et la pression sociale qu'institue une société via ses normes et conventions n'a été étudiée que de manière insuffisante jusqu'ici. Mais les études comparées internationales fournissent un certain nombre d'indices montrant qu'il est plus que vraisemblable que la pression intégratrice qu'exerce une société est d'autant plus forte que les contraintes institutionnelles émanant de l'Etat sont faibles. La liberté (ou libéralisme) politique ne 
mènerait donc pas, contrairement à ce qu'on pourrait être tenté de croire, à la dissolution des normes et valeurs, une menace pour la cohésion sociale, mais bien au contraire, elle favoriserait la cohésion sociale, même si elle le fait au prix d'une pression plus forte pesant sur chaque individu et le forçant à s'intégrer. Cette pression pourrait se manifester par exemple dans l'intolérance croissante envers les opinions et comportements qui divergent de la norme communément acceptée par une société.

Pour se faire une au moins première idée, inévitablement superficielle, du degré de tolérance dans la société allemande, l'institut IfD a posé à l'été 2011 une série de questions sur la liberté d'opinion - au sens général et non pas sous l'angle juridique. Ces questions n'avaient donc pas pour objet la garantie constitutionnelle de la liberté d'expression, mais cherchaient à mesurer la tolérance des Allemands vis-à-vis d'opinions n'étant pas « politiquement correctes » et leur perception subjective de la possibilité d'exprimer de tels points de vue sans craindre une sanction de la part de leurs concitoyens.

Ces questions visent à déterminer d'une part quelles sont les assertions « politiquement incorrectes » que les personnes interrogées tolèrent; d'autre part, quelles sont celles que les sondés considèrent majoritairement comme si insupportables qu'ils pensent qu'il devrait être interdit de dire de telles choses, et enfin quelles sont celles dont ils croient qu'elles leur attireraient des ennuis s'ils les prononçaient. II faut se garder de confondre ces deux derniers types de questions : l'une (demande d'interdit) cherche en effet à mesurer l'intolérance effective de la société envers un certain nombre d'assertions ; l'autre (le fait de croire que c'est risqué) cherche à déterminer le climat de l'opinion publique - or celui-ci n'est pas nécessairement identique avec la répartition des diverses opinions dans la population.

Comme il était probable que les questions sur l'intolérance réelle et l'intolérance ressentie risquaient de s'influencer mutuellement, et ainsi de fausser les réponses, si on les soumettait à la même personne interrogée puisque celle-ci chercherait à répondre avec le moins de contradictions possibles, I'IfD a choisi une démarche expérimentale pour cette partie de l'enquête. Ainsi, à la première moitié des sondés a été remis un jeu de cartes. Sur chaque carte figurait une assertion contraire aux valeurs morales prédominantes et dont on pouvait supposer qu'elle serait rejetée, souvent vivement, par la plupart des sondés. On leur a ensuite demandé de classer ces assertions en trois catégories : celles avec lesquelles ils sont d'accord; celles avec lesquelles ils ne sont pas d'accord mais dont ils estiment qu'il doit être permis de les prononcer ; enfin, celles dont ils pensent qu'il devrait être interdit de dire cela. On a remis un jeu de cartes avec les mêmes assertions à l'autre moitié des sondés. Mais au lieu de leur demander leur propre opinion, on leur a posé la question suivante : "Tout le monde sait qu'il y a des choses qui choquent quand on les dit en public. Sur ces cartes sont écrits certains de ces propos. Pour lesquels d'entre eux diriezvous qu'ils sont particulièrement scabreux et qu'on court le risque de se faire taper sur les doigts en disant une chose pareille ? ॥

Le graphique 5 montre les réponses aux deux types de questions. La première remarque qui s'impose est que, dans la société allemande, l'intolérance envers les opinions entrant en condradiction avec l'idée que chacun se fait d'un comportement respectueux des valeurs morales est extraordinairement élevée. Sur le total des 22 assertions soumises aux sondés, deux recueillent une nette majorité, et dix une importante minorité de réponses plaidant pour les interdire. Certes, il est compréhensible qu'une majorité de $58 \%$ des Allemands juge que les propos négationnistes devraient être interdits. Mais au vu de l'histoire allemande et du droit spécifique en la matière (en Allemagne, les propos négationnistes relèvent du délit pénal d'incitation à la haine raciale), on peut affirmer qu'il s'agit ici d'un cas particulier. II en va tout autrement de la formule "Les soldats sont des assassins " ("Soldaten sind Mörder») qu'une majorité d'Allemands souhaiterait voir interdire.
Quelle perception subjective de la liberté d'expression?

Intolérance réelle et intolérance ressentie

Une méthode expérimentale pour mesurer l'écart

Une intolérance forte dans le ressenti 
Intolérance surtout vis-à-vis des opinions qu'on ne partage pas
Les réponses révèlent par ailleurs que c'est moins le caractère plus ou moins radical d'une assertion qui détermine la réaction de demander son interdiction que son caractère plus ou moins éloigné de l'opinion personnelle de la personne interrogée. Schématiquement, cela revient à dire que quelqu'un qui se situe politiquement à gauche souhaite voir interdire des assertions marquées à droite et vice et versa. L'idée même que le droit fondamental de la liberté d'expression inscrit dans la Constitution s'applique également aux opinions marginales ou moralement peu défendables, semble à l'évidence ne plus effleurer l'esprit de beaucoup d'Allemands.

\section{"Soldaten sind Mörder»}

Cette phrase, une des plus célèbres citations des temps modernes, est tirée d'un billet écrit par le journaliste et écrivain humaniste et pacifiste Kurt Tucholsky et publiée en 1931 dans la revue Die Weltbühne; elle a depuis mobilisé les tribunaux à intervalles réguliers. Dans la RFA des années 1980, elle était devenue le slogan des différents mouvements pacifistes et antimilitaristes allemands et avait donné lieu à une série de condamnations pour incitation à la haine de ceux qui l'avaient prononcée en public ou publiée, à l'instar du magazine satirique Titanic.

Le Tribunal constitutionnel, saisi à trois reprises, a alors rendu en 1995 un arrêt de fond très controversé, dans lequel il rappelait l'étendue de la liberté d'expression : celle-ci reste infrangible même lorsque sont prononcés des jugements collectifs (y compris des caricatures) portant atteinte à l'honneur d'un groupe ou corps de la société, en l'occurrence l'armée. La liberté d'opinion garantie par la Constitution autorise donc que soit prononcée la phrase «Les soldats sont des assassins »; le droit constitutionnel interdit donc de l'interdire. (IB)

\section{Graphique 5a}

Tabous existants et tabous ressentis I

Questions :

"Sur ces cartes sont écrits des propos sur lesquels les opinions peuvent diverger. Veuillez distribuer ces cartes sur cette image en les classant selon que vous pensez que ce qui est écrit est exact et que vous partagez cette opinion ou bien selon que vous n'êtes pas d'accord, mais que vous estimez qu'on ne peut interdire à personne de dire cela, ou encore selon qu'à votre avis il devrait être interdit de dire de telles choses " (présentation des cartes et de l'image).

"Tout le monde sait qu'il y a des choses qui choquent quand on les dit en public. Sur ces cartes sont écrits certains de ces propos. Pour lesquels d'entre eux diriez-vous qu'ils sont particulièrement scabreux et qu'on court le risque de se faire taper sur les doigts en disant une chose pareille? " (présentation du jeu de cartes).

- Il devrait être interdit de dire cela nOn peut se faire taper sur les doigts en disant ça

Ce qu'on rapporte sur les camps de concentration est exagéré

Les soldats sont des assassins

On devrait arrêter de payer les opérations des personnes âgées

Il faudrait réintroduire la peine de mort

L'homosexualité est une maladie

Le 11 septembre est de la faute des Américains

Il faudrait reconstruire le Mur

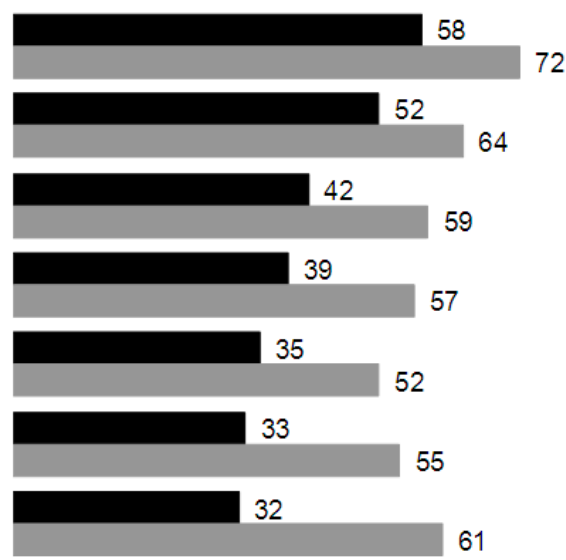

Source : Allensbacher Archiv, sondage IfD-Umfrage $n^{\circ}$ 10077, août 2011.
Tabous réels et tabous ressentis
Le deuxième constat digne d'être relevé appert quand on compare les tabous existants et les tabous "ressentis". On peut penser dans un premier temps que les opinions qu'un nombre important de personnes interrogées voudraient voir interdire sont les mêmes que celles dont la plupart pensent qu'on « se fait 
taper sur les doigts » si on les exprime publiquement. Mais cela n'est vrai qu'en partie.

II existe certes des affirmations comme les propos négationnistes ou la thèse "les soldats sont des assassins " que beaucoup de personnes veulent voir interdire et qu'elles ressentent également comme risquées à dire publiquement. Et il existe aussi un certain nombre d'assertions qui ne choquent que peu d'Allemands et que peu d'entre eux auraient peur de formuler publiquement, par exemple " ce qu'on nous raconte sur le réchauffement climatique est exagéré, le réchauffement climatique n'existe pas » ou "l'intelligence est héréditaire $"$.

\section{Graphique 5b}

Tabous existants et tabous ressentis II

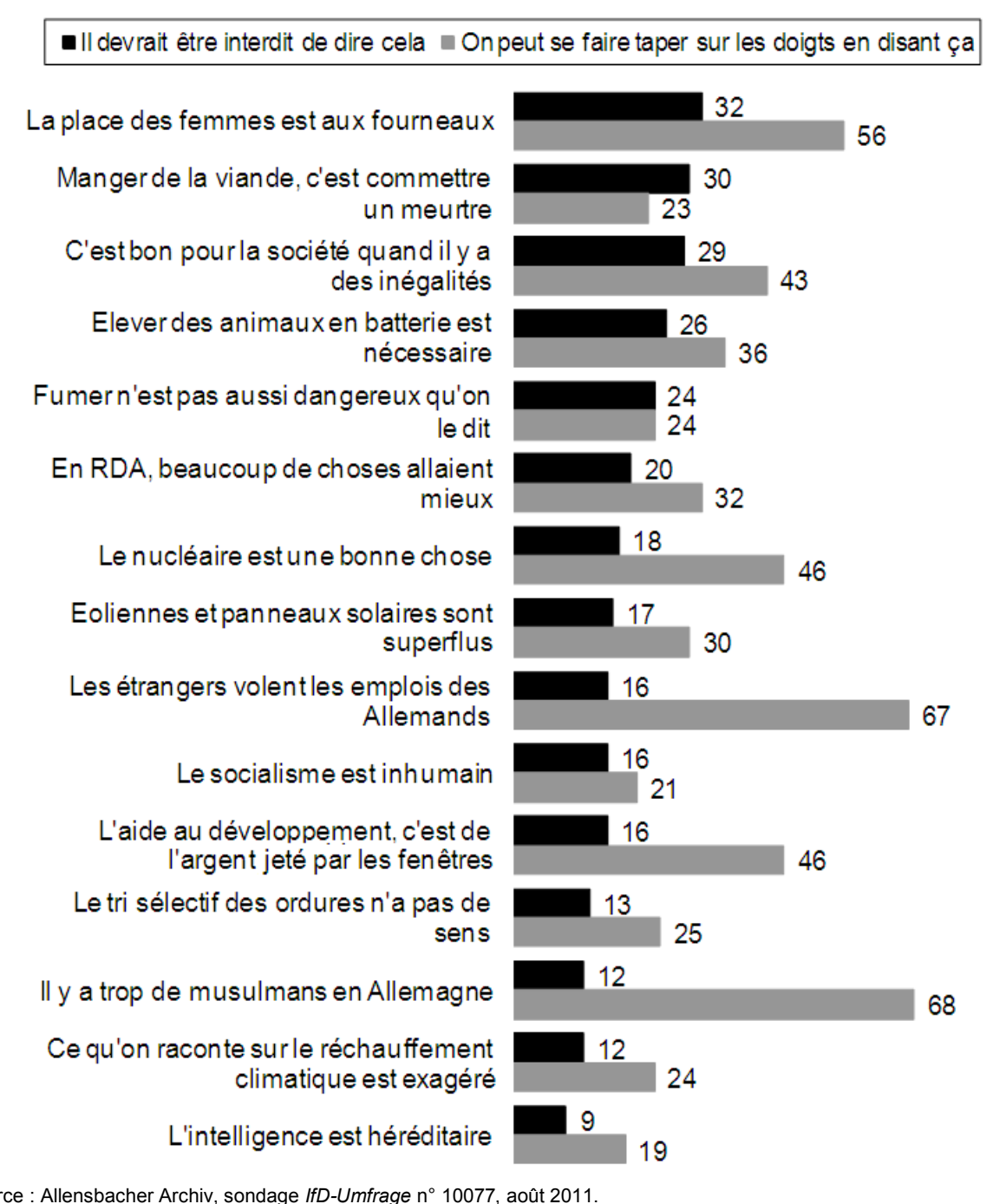

Source : Allensbacher Archiv, sondage IfD-Umfrage n 10077, août 2011.

Mais il y a aussi un certain nombre de cas où l'intolérance effective de la Le rôle déterminant des médias société et l'impression qu'on risque de se brûler les doigts divergent profondément. C'est particulièrement vrai de l'affirmation : " Il $y$ a trop de musulmans en Allemagne », où " l'intolérance ressentie » est cinq fois plus importante que l'intolérance réelle. Sur certains points, tout particulièrement lorsqu'ils touchent l'opinion des sondés sur les immigrés, on constate donc un écart étonnamment important entre l'état réel de l'opinion publique et le climat de l'opinion tel qu'il est ressenti. Or comme ce ressenti ne peut pas provenir de la seule observation de l'environnement immédiat des personnes interrogées, parce qu'alors, il n'y 
aurait pas un tel écart, c'est dans l'autre source principale de la formation de l'opinion publique et politique qu'il convient de chercher l'explication de cette profonde divergence : à savoir dans l'information par les médias de masse. Et de fait, le schéma qu'on observe ici est typique pour les situations où le jugement porté par les médias diffère des appréciations existant dans l'opinion publique. Les thèmes concernés méritent la plus haute attention car la plupart du temps, la forte pression exercée par les médias sur le climat de l'opinion masque des conflits sociétaux non résolus.

ON S'APERÇOIT FINALEMENT, au vu de tous ces résultats, que la valeur que les Allemands accordent à la liberté est empreinte de toute une série de contradictions, et qu'ils n'en sont pas toujours conscients. Alors que, ces dernières années, les Allemands ont développé de manière croissante une approche foncière de la vie sur laquelle se fonde leur approbation de la liberté et la valeur qu'ils lui accordent, dans le même temps augmente dans la population la part de ceux qui considèrent avec méfiance le principe d'une économie libérale. Par ailleurs, les citoyens exigent de l'Etat de plus en plus de contrôles, d'interdits et d'interventions dans les domaines les plus divers de leur vie. Or le constat qu'un nombre croissant d'interdits s'accompagne automatiquement d'une limitation de la liberté individuelle est tout sauf anodin. Car visiblement, dans leur quotidien, nombreux sont les Allemands qui n'ont pas conscience de cette implication.

Les Allemands sont nombreux aussi à avoir développé une forte intolérance envers tout propos contraire à leurs propres convictions. Et de larges minorités d'entre eux vont jusqu'à revendiquer l'interdiction de grand nombre d'opinions exprimées. II semble que, souvent, la liberté d'expression est acceptée ou rejetée en fonction du cas particulier rencontré par les sondés dans la vie réelle. II n'est pas infondé de supposer que le même relativisme s'applique aussi à tous les autres domaines de la vie où ils peuvent être appelés à choisir pour ou contre la liberté. Tout porte donc à croire que l'idée même que la liberté est un principe universel régissant l'organisation de la société et que ce principe vaut pour tous les citoyens pourvu qu'ils respectent la loi, n'effleure plus guère l'esprit de nombre d'Allemands. Pour beaucoup d'Allemands, la liberté semble bien plutôt se réduire à celle qu'on partage avec ceux qui pensent comme vous.

De tels constats ne devraient pas sembler étrangers à nombre de lecteurs français - et c'est là peut-être le principal enseignement que nous livrent ces sondages. Aux yeux d'un Français, les Allemands sont souvent difficiles à cerner, tant ils lui semblent étrangement disciplinés, sachant garder la tête froide et se comporter rationnellement. Mais ce n'est là qu'apparence. Et peut-être est-ce là aussi qu'il faut chercher une des raisons profondes qui, malgré tous les problèmes actuels, expliquent le remarquable succès de l'unité européenne et plus encore du partenariat franco-allemand au cours des dernières décennies. Car en réalité, et sous de nombreux aspects, les Allemands ressemblent beaucoup plus aux Français qu'ils ne le soupçonnent. Ils vont même, quand ils entonnent leur hymne national, jusqu'à chanter des paroles françaises... A lui seul, ce fait est étonnant. Mais ce qui est bien plus significatif encore, c'est que les Allemands le font sans même s'en apercevoir.

(Traduction : I. Bourgeois) 


\section{Indications bibliographiques}

- BourgeoIS I., « Opinion : les valeurs „bourgeoises“, ciment de la société allemande », Regards sur l'économie allemande, $\mathrm{n}^{\circ} 84$, décembre 2007

- BouRgeoIs I., " Opinions et valeurs : 60 ans de démoscopie allemande », Regards sur l'économie allemande, $\mathrm{n}^{\circ} 80$, mars 2007

- ChURChland P., « Vernunft braucht Gefühle », in MAAR C., PöPPEl E., Christaller T. (eds), Die Technik auf dem Weg zur Seele. Forschungen an der Schnittstelle Gehirn/Computer, Hambourg, 1996

- GÄRTNeR W., « Justice, productivité, efficience et contexte culturel », Regards sur l'économie allemande, $\mathrm{n}^{\circ} 59$, décembre 2002

- GRIMM J., GRIMm W., Deutsches Wörterbuch, vol. 4, $1^{\text {ère }}$ partie, Leipzig, 1878/Munich, 1984

- GWARtney J., Hall J., LAWSON R., Economic Freedom of the World 2010. Annual Report. Vancouver, Fraser Institute, 2010

- Huntington S. P., "Will More Countries Become Democracies ? ", in Political Science Quarterly, $\mathrm{n}^{\circ}$ 99, 1984

- KEPPLinger H. M., «Wirkung der Massenmedien », in NOELle-NeUmanN E., SCHULZ W., WILKE J. (eds), Fischer Lexikon Publizistik Massenkommunikation, Francfort/Main, 2009

- LUTHER M., «Von der Freiheit eines Christenmenschen » [1520], in LUTHER M., Von der Freiheit eines Christenmenschen. Fünf Schriften aus den Anfängen der Reformation (Edition Calwer Luther-Ausgabe, vol. 2), Neuhausen auf den Fildern, 1996

- Miller T., HOLmes K. R., FEULner E. J., Highlights of the 2012 Index of Economic Freedom. Promoting Economic Opportunity and Prosperity, The Heritage Foundation, Washington, 2012

- NOELLe-Neumann E., Die Schweigespirale. Öffentliche Meinung - unsere soziale Haut, Munich, 2001

- Petersen T., «Frères et soeurs dissemblables. Un bilan de l'Unité allemande » Regards sur l'économie allemande, $\mathrm{n}^{\circ}$ 98-99, décembre 2010

- Petersen T., Das Feldexperiment in der Umfrageforschung, Francfort/Main, 2002

- Petersen T., PR-Arbeit in der Antike. Wie Augustus zum vielleicht erfolgreichsten Politiker aller Zeiten wurde, Munich, 2005

- Petersen T., « Der geteilte Liberalismus », Frankfurter Allgemeine Zeitung, 25-01-2012

- Petersen T., MAYeR T., Der Wert der Freiheit. Deutschland vor einem neuen Wertewandel ?, Fribourg, 2005

- REIN G., «Das verbotene Jenseits. Gespräch mit Max Horkheimer kurz vor dessen Tod », Weltwoche, 25-07-1973

- TocqueVille A. DE, De la démocratie en Amérique, tome 1, Paris, 1991. 